\title{
Elastohydrodynamic Films Under Periodic Load Variation: An Experimental and Theoretical Approach
}

\author{
Romeo Glovnea ${ }^{1}$ (D) $\cdot$ Xingnan Zhang ${ }^{1}$
}

Received: 26 April 2018 / Accepted: 24 July 2018 / Published online: 6 August 2018

(c) The Author(s) 2018

\begin{abstract}
The elastohydrodynamic lubrication regime occurs in systems where large elastic deformations, the hydrodynamic action of a converging wedge and eventually large variation of viscosity of the fluid combine to determine the formation of a continuous fluid film that separates the solid surfaces. Experimental and theoretical works, over the past few decades, have elucidated the role of various working and material parameters on the lubricant film thickness which plays a crucial role in protecting the solid surfaces from direct contact and ultimately from failure. These mechanisms are well understood for steady-state conditions; however, elastohydrodynamic contacts most often experience transient conditions, including variation of geometry, velocity of surfaces or load. In this case, the mechanisms of film formation are more complex involving film squeeze in addition to the mechanisms mentioned above. Experimental and theoretical modelling of transient phenomena in elastohydrodynamic lubrication include sudden variation of entrainment speed or load and changing geometry. No systematic experimental study on the effect of harmonic load vibration upon the elastohydrodynamic films has been published before. In order to cover this gap, this paper presents the results of an experimental study and of a simple theoretical approach on the behaviour of the elastohydrodynamic film thickness under harmonic variation of load.
\end{abstract}

Keywords Elastohydrodynamic $\cdot$ Film thickness $\cdot$ Vibrations $\cdot$ Harmonic force

\section{Introduction}

The formation of elastohydrodynamic (EHD) films, in steady-state conditions, is governed by the hydrodynamic action of the lubricant forced into a converging conjunction, the elastic flattening of the contacting surfaces and the variation of lubricant's viscosity with pressure. A theoretical, semi-analytical study, applied to linear contacts, by Ertel and Grubin $[1,2]$ and published by Grubin and Vinogradova, showed how these mechanisms contribute together to result in a parallel lubricant film separating the solid surfaces. The equation for the film thickness derived by Ertel/ Grubin not only revealed the physics of the phenomena but proved to be very close to more precise, later numerically derived formulas.

This important feature of a parallel lubricant film, over most part of the central region of the contact, was proved by

Romeo Glovnea

R.P.Glovnea@sussex.ac.uk

1 Department of Engineering and Design, University of Sussex, Brighton BN1 9QT, UK many experimental results starting with those published by Gohar and Cameron [3, 4] who used optical interferometry to observe the film shape and measure the film thickness in circular, point contacts. The principles of optical interferometry, as applied to the study of EHD lubrication will be explained in a later section. An example of an image of a point contact is shown in Fig. 1 captured by optical interferometry for PAO 40 (poly-alpha-olefin) oil, at an entrainment speed of $0.126 \mathrm{~m} / \mathrm{s}$, under $20 \mathrm{~N}$ normal contact load and at ambient temperature.

As seen, for most part of the central region of the contact the colour is uniform indicating a constant film thickness. This is because over that region the pressure is very large and varies little making the lubricant film almost unchanged until it reaches the outlet area of the contact, where a constriction in the film thickness occurs. The constriction, which also occurs at the sides of the contact, is a result of the rapid decrease of the pressure at the outskirts of the contact which must be accompanied by a change in film thickness in order to satisfy the pressure gradient condition imposed by Reynolds equation [2]. This feature is not included in Ertel-Grubin analysis, although they were aware 


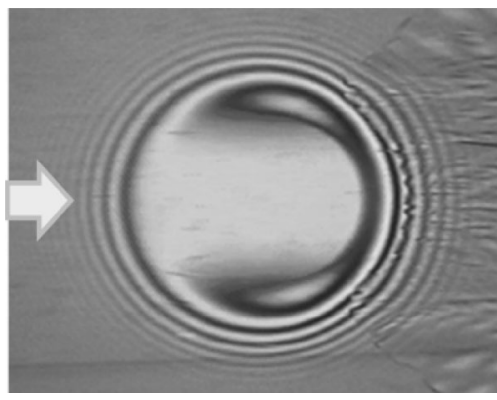

Fig. 1 Example of contact image by optical interferometry

of the phenomenon and discussed it. Later, Greenwood [5] derived the exit constriction theoretically by moving the parallel region of the film off centre.

Early theoretical approaches of the lubricant film thickness in EHD contacts also include the works of Archard and Kirk [6] and Cameron and Gohar [7] focused on point rather than line contacts; however, the real breakthrough in obtaining a reasonably accurate lubricant film thickness formula is due to Dowson and Higginson [8]. They solved numerically the coupled equations of the pressure in front of the contact (Reynolds), the film shape in that region (Hertz) and the variation of the lubricant's viscosity with pressure (Barus) to obtain an equation for film thickness for line contacts supported well by later experimental results. Later on, Dowson and co-workers $[9,10]$ have used the same method to solve the elliptical contact film thickness problem. A widely used relationship of the central film thickness in elliptical contacts is due to Hamrock and Dowson [9] and seen in Eq. (1).

$\frac{h_{\mathrm{c}}}{R_{x}}=2.69 \bar{U}^{0.67} \bar{G}^{0.53} \bar{W}^{-0.067}\left(1-0.61 e^{-0.73\left(R_{y} / R_{x}\right)^{0.64}}\right)$.

In this equation $\bar{U}, \bar{G}$ and $\bar{W}$ are, respectively, the speed, material and load non-dimensional parameters given by

$\bar{U}=\frac{U \eta_{0}}{E^{*} R_{x}}, \quad \bar{G}=\alpha E^{*}, \quad \bar{W}=\frac{W}{E^{*} R_{x}^{2}}$

(U-entrainment speed, that is average speed of the surfaces in the direction of rolling, $x, \eta_{0}, \alpha-$ viscosity and pressure-viscosity coefficient of the lubricant at ambient pressure, $W$-load, $E^{*}$-reduced elastic modulus of the solids, and $R_{x}, R_{y}$-the reduced radii of curvature in the direction of entrainment and perpendicular to it, respectively). Equation (1) shows that the parameters with the strongest influence upon the film thickness are the entrainment speed and the lubricant's viscosity. On the opposite side is the load which has the weakest effect. This is due to the increase of lubricant's viscosity with pressure and the fact that once inside the contact the lubricant cannot escape easily making the lubricant film stiffer than the solid, surrounding surfaces. Equation (1) was validated by experimental results as shown in many publications [11-13].

It can be said that the formation of elastohydrodynamic films and the lubricant film thickness are well understood in steady-state conditions, when the velocity, load and geometry remain unchanged with time. In real-life applications however, steady state is rarely obtained in machine components working in EHD regime of lubrication. For some of these machine components, like gears and cams the causes of non-steady conditions are first of all intrinsic and result from their geometry and kinematics, which imply large variations of sliding/rolling velocities and of the load. In others, like rolling element bearings steady-state conditions can be achieved when they run at constant speed and under constant load; however, transient speed conditions always occur at start and stop of the motion while transient loading is the result of vibrations which are almost inevitably transmitted through the bearing.

Research into the behaviour of elastohydrodynamic films in transient conditions has received merited attention during the relatively recent past, with the focus mainly on geometry and speed variation. The research onto the transient effect of geometry upon the behaviour of EHD films has been driven by studies on mixed lubrication, where roughness plays an important role [e.g. 14-16] and those on surface featured lubrication seen as a way to improve load carrying capacity and reduce friction drag [17-19].

A good deal of papers on transient effects in EHD lubrication considered the behaviour of the lubricant film under rapid variation of velocity of surfaces, in both experimental and numerical approaches. These studies have shown that the transit of the lubricant through the contact, under variable entrainment speed, causes fluctuations of the film thickness as any perturbation generated in the inlet travels at the average speed of the surfaces [20,21]. Under sudden halting of motion, the rapid film decrease is delayed by a squeeze effect eventually leading to fluid entrapments formed inside the contact [22,23]. Conversely, when the surfaces are suddenly set in motion, oscillations with decreasing amplitude are generated in the film thickness due to the instability created in the inlet of the contact $[24,25]$. Similar fluctuations of the film thickness were observed during a step load of the contact [26-28]. This points to the conclusion that the elastohydrodynamic film and the surrounding, solid bodies form a mass, spring damper, dynamic system which responds to a sudden shift from equilibrium in a manner known from vibrations theory [29].

Apart from the sudden application of load, studies concerning the effect of load variation upon the behaviour of EHD films are rare, due probably to the fact that the load has small influence upon the steady-state film thickness and the relatively difficult experimental conditions. Kilali et al. 
[30] used an optical-based experimental rig combined with a system which superimposed a normal load excitation on the static one to measure the fluid film thickness and the dynamic response of the contact subjected to a harmonic loading. Limited results are presented and none on the response of the film under harmonic vibrations. Sakamoto et al. [31] also applied the optical interferometry technique to directly observe the effects of cyclic pulsating and impact load on EHD point contact under static and rolling/sliding conditions. They observed an oil entrapment formed during rapid increase of the load. The thickness of this entrapment is diminished as the overall entrainment speed of the contact increases.

Morales-Espejel [32], Felix-Quinonez and MoralesEspejel [33] proposed an improved semi-analytical method for evaluating the film thickness fluctuations in the normal-approach of EHD contacts. The method was based on squeeze film effects as well as the variation of lubricant entrainment speed caused by the oscillating load and change of the conditions in the inlet region of the EHD contact. Film thickness results predicted by their semi-analytical method were compared with full numerical simulations. It was concluded that film thickness perturbations could be ascribed to two effects: squeeze film phenomenon caused by vertical rigid body motion of the surfaces and variation in effective entrainment velocity due to fluctuations of contact width.

In the present research, the authors conducted experiments on the measurement of the elastohydrodynamic film thickness in contacts subjected to harmonic variation of load. The behaviour of the EHD film was evaluated and, on that basis, a qualitative theoretical analysis was devised and carried out.

\section{Experimental Setup and Working Conditions}

The EHD film thickness was measured by the optical interferometry technique, described in detail by these and other authors in various publications [34-36]. The schematic of the experimental arrangement is shown in Fig. 2. An EHD contact was formed between the flat surface of a glass disc and a highly polished steel ball. The disc is driven at desired speed by a shaft coupled to an electric motor. The ball is half-immersed in the lubricating fluid and driven by the shear forces developed in the EHD film, resulting in a nominally pure rolling contact. The ball is supported by a shaft which in turn is fitted to a carriage providing bearings support. The ball carriage is attached to a steel bellow, which ensures that vertical displacement can be applied to the ball and that the lubricant does not leak from the lubricant chamber, and a plunger which allows the load to be transmitted to the ball.

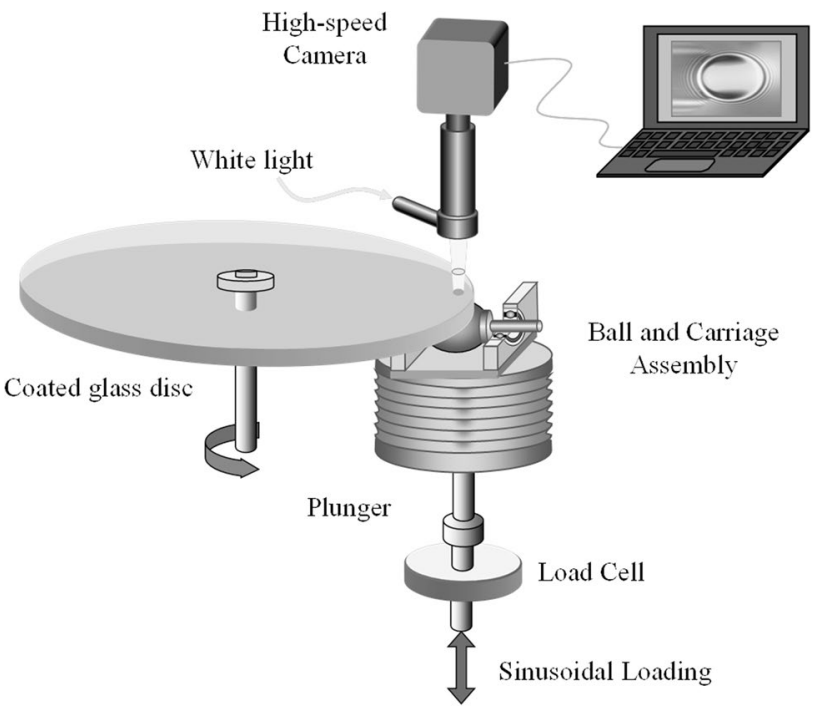

Fig. 2 Schematic of the experimental setup

In between a lever which oscillates under the action of a dynamic shaker and the plunger attached to the bellow, a load cell was placed such that the force applied directly to the contact was known. A waveform generator was used to provide a true sinusoidal signal for the shaker. The electrical signal from the load cell was amplified and sent to a digital oscilloscope for visualisation and recording. The dynamic response of the test rig is important in such experiments where some of the parts are subjected to oscillatory motion. For this reason, the rig was designed to be as stiff as possible, given the space limitations. The shaft supporting the ball was made with a relatively large diameter, while the bearings supporting this shaft were placed very close to the ball. Moreover, needle bearings were chosen to support the shaft as their linear contacts make them considerably stiffer than ball bearings. In order to minimise the deflection of the glass disc, this was made relatively thick $(12 \mathrm{~mm})$ and was also supported by an additional, rolling support diametrally opposite to the location of the studied contact; this prevented the deflection of the disc' shaft. Exact modelling of the dynamic response was not one of the objectives of the paper; however, a simplified analysis was carried out, which showed that the natural frequency of the test rig is over $2100 \mathrm{~Hz}$, thus well beyond the maximum frequency employed in these experiments, that is, $100 \mathrm{~Hz}$.

The contact was illuminated through the transparent glass disc, which was coated with a thin, semi-reflective chromium layer (5-10 nanometres) and an aluminium oxide layer (approximately 150 nanometres) on the contacting surface. The former was to provide amplitude division of the light waves of a white source while, the latter was to increase the gap between the two reflective surfaces, that is, chromium and steel. The light rays reflected by these 
surfaces recombine and interfere constructively or destructively depending of their particular wavelengths resulting in a coloured image of the EHD contact, as seen in Fig. 1. These images were captured by a high-speed CCD camera and sent to a laptop computer for storage and analysis. A simple electrical circuit ensured that the camera and oscilloscope recordings were synchronised such that the value of the force loading the contact at each frame time of the camera was precisely known. Details of this arrangement are provided in [37].

The research encompassed a larger number of lubricants, but the results shown in this paper are for a poly-alpha-olefin synthetic lubricant, PAO40, with kinematic viscosity of $339.8 \mathrm{~mm}^{2} / \mathrm{s}(339.8 \mathrm{cSt})$ at $40{ }^{\circ} \mathrm{C}$ and $35.8 \mathrm{~mm}^{2} / \mathrm{s}(35.8$ cSt) at $100{ }^{\circ} \mathrm{C}$. The density at $40{ }^{\circ} \mathrm{C}$ is $840 \mathrm{~kg} / \mathrm{m}^{3}$. Because $\mathrm{PAO} 40$ is a viscous lubricant, it forms a thick film even at low entrainment speeds, and thus the results analysed in this paper were obtained at the lowest entrainment speed of the tests of $50 \mathrm{~mm} / \mathrm{s}$ and ambient temperature of $21 \pm 1{ }^{\circ} \mathrm{C}$.

The load applied was varied harmonically between about 1-4 $\mathrm{N}$ minimum and 43-56 $\mathrm{N}$ maximum, at frequencies of 10, 25, 50 and $100 \mathrm{~Hz}$. This gives a Hertzian pressure variation, for steel on glass, of between approximately 0.2 and $0.7 \mathrm{GPa}$ (the steel ball is $19.05 \mathrm{~mm}$ diameter super-finished at $10-15 \mathrm{~nm} \mathrm{Ra}$ ). Thousands of cycles of load variation were recorded for each test, showing excellent consistency of the load amplitude in each of these tests. There were variations of the amplitude between tests especially at the largest frequency of $100 \mathrm{~Hz}$. The shaker oscillates in an alternating cycle, while the load variation required was pulsating. In order to deal with this, a constant load was initially applied and with the shaker in operation, this load was adjusted to give the desired load variation to the contact. This was a delicate operation, and thus it was not always possible to adjust the load exactly between zero and the maximum value. Complications also resulted from the fact that the amplitude of the oscillations of the shaker decreased with the increase of the frequency. The large number of repetitions of each test condition and number of cycles recorded allowed enough good data to be saved for analysis. Table 1 summarises all the operating conditions for different conducted experiments.

\section{Experimental Results}

Figures 3, 4, 5 and 6 show the load variation, at four different frequencies and a number of seven interferometry images of the contact selected to cover one cycle of load variation. The black dots on each of the load-variation cycles correspond to the time location of the selected images within the cycle. The time-stamp for each image as well as the direction of the entrainment motion are shown on the images. As seen, the load varies on a fashion that approximates well a sinusoid curve.

It is well known that in white light interferometry various colours signify variation of the gap between the surfaces, thus different film thicknesses of the lubricant film. It is thus immediately noticed from the images shown that, for all frequencies, the film thickness is different in the load-increasing and load-decreasing phases. It is also obvious from these images that the EHD film behaves differently depending on the frequency of the load variation. For the lowest frequency, that is $10 \mathrm{~Hz}$, the typical EHD film image is shown with a large, central area of nearly constant thickness; however, at the largest two frequencies, 50 and $100 \mathrm{~Hz}$ rings of different colours are seen inside the contact, indicating relatively large variation of the film thickness within the contact area. The reasons for this behaviour will be discussed in the following section.

\section{Discussion}

\subsection{Experimental Observations}

The interferometry images shown in figures above were converted into film thickness maps according to a calibrations procedure which relates the RGB colour content in every pixel of the image to a lubricant film thickness value. Details of the calibration procedure and an accuracy analysis are presented in [37]. Figure 7 shows the variation of the load and central film thickness over one cycle, for the four frequencies employed in these experiments. In all these, the entrainment speed set was $0.05 \mathrm{~m} / \mathrm{s}$.

Table 1 Test conditions of experiments

\begin{tabular}{|c|c|c|c|c|c|}
\hline Test lubricant & Kinematic viscosity & Temperature & Entrainment speed $(\mathrm{m} / \mathrm{s})$ & Frequency $(\mathrm{Hz})$ & $\begin{array}{l}\text { Load } \\
\text { amplitude } \\
\text { (N) }\end{array}$ \\
\hline \multirow[t]{4}{*}{ PAO 40} & \multirow{4}{*}{$\begin{array}{l}339.8 \mathrm{~mm}^{2} / \mathrm{s}(339.8 \mathrm{cSt}) \text { at } 40^{\circ} \mathrm{C} \\
35.8 \mathrm{~mm}^{2} / \mathrm{s}(35.8 \mathrm{cSt}) \text { at } 100{ }^{\circ} \mathrm{C}\end{array}$} & \multirow{4}{*}{$\begin{array}{l}\text { Ambient } \\
21 \pm 1{ }^{\circ} \mathrm{C}\end{array}$} & \multirow[t]{4}{*}{0.05} & 10 & $1-52$ \\
\hline & & & & 25 & $0.7-53$ \\
\hline & & & & 50 & $0.6-56$ \\
\hline & & & & 100 & $2-43$ \\
\hline
\end{tabular}


Fig. 3 a Example of load variation, $10 \mathrm{~Hz}$, PAO 40 . b. Interferograms of the PAO 40 at ambient temperature, captured at $10 \mathrm{~Hz}$

Fig. 4 a Example of load variation, $25 \mathrm{~Hz}, \mathrm{PAO} 40$. b. Interferograms of the PAO 40 at ambient temperature, captured at $25 \mathrm{~Hz}$

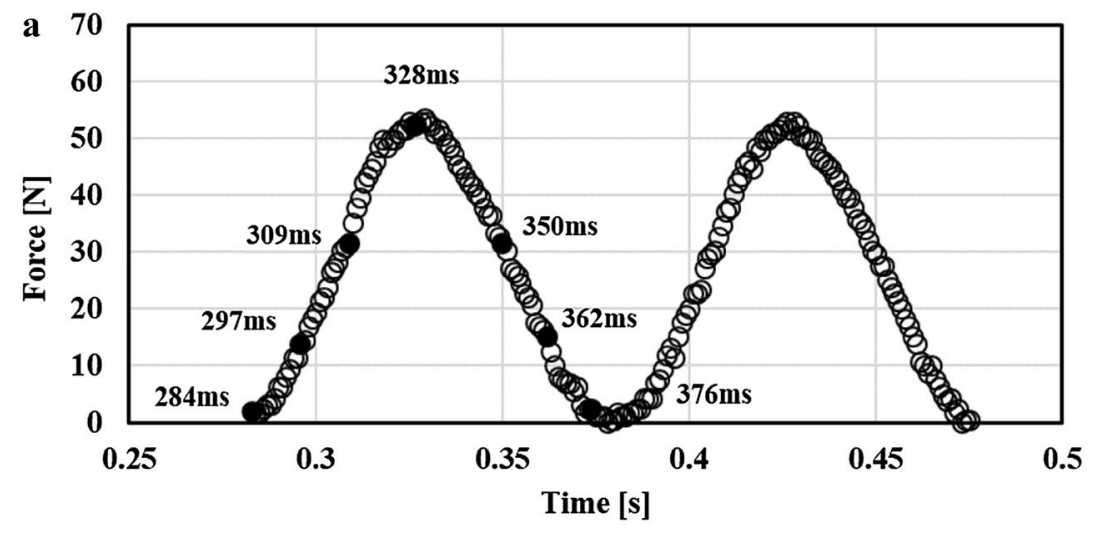

b
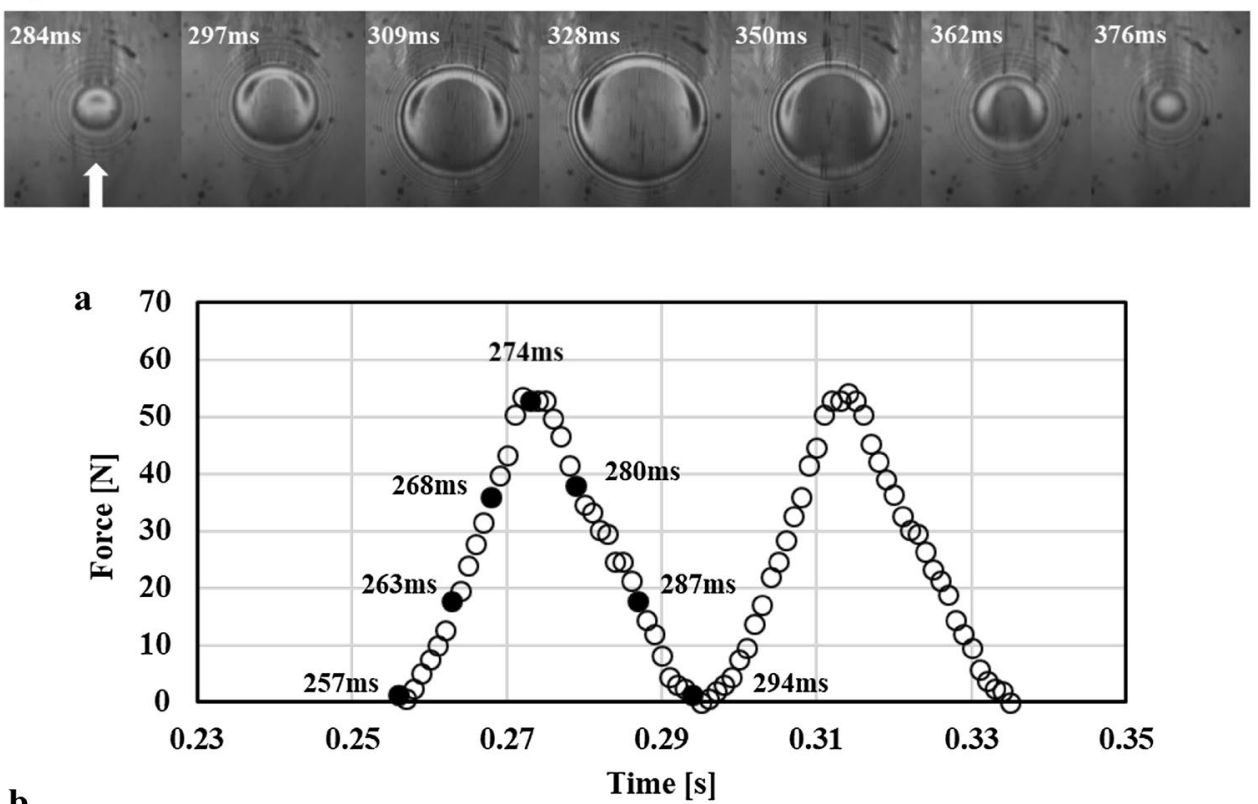

b

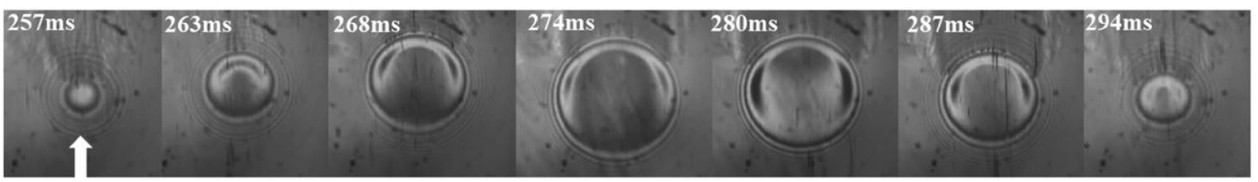

Given the fact that the load variation is approximately symmetric in the increasing and decreasing phases, it would be expected that the film thickness variation is also symmetric, which as seen is not the case. For the larger frequencies, the central film thickness is much larger during the loadincreasing than in the load-decreasing phase. This is better observed in Fig. 8 which shows the relative variation over one cycle. The theoretical film thickness, calculated from the measured load and entrainment speed, is also shown. $T$ is the period of the given cycle of load variation. As seen, there is a strong difference of the behaviour of the elastohydrodynamic film depending of the frequency of the load variation.

The theoretical film thickness [Eq. (1)] varies little with load, as expected, even if this parameter changes more than 15 times; however, the experimental values show large variation over the load cycle at the largest frequency of $100 \mathrm{~Hz}$.

At the beginning of the load-increasing phase, the measured film thickness is about $65 \%$ larger than the theoretical, steady-state value. It drops continuously later and becomes smaller (about 95\%) when the load approaches the minimum value in the load-decreasing phase. At $50 \mathrm{~Hz}$, the transient experimental film thickness is just under $40 \%$ larger than the steady-state value, at the beginning of the load-increasing phase and about $20 \%$ lower when the load reaches the lowest value of the cycle. At the two lowest frequencies 10 and $25 \mathrm{~Hz}$, the experimental values follow the theoretical one, within the error of the method, over the whole cycle. 
Fig. 5 a Example of load variation, $50 \mathrm{~Hz}, \mathrm{PAO} 40$. b Interferograms of the PAO 40 at ambient temperature, captured at $50 \mathrm{~Hz}$

Fig. 6 a Example of load variation, $100 \mathrm{~Hz}, \mathrm{PAO} 40$. b Interferograms of the PAO 40 at ambient temperature, captured at $100 \mathrm{~Hz}$
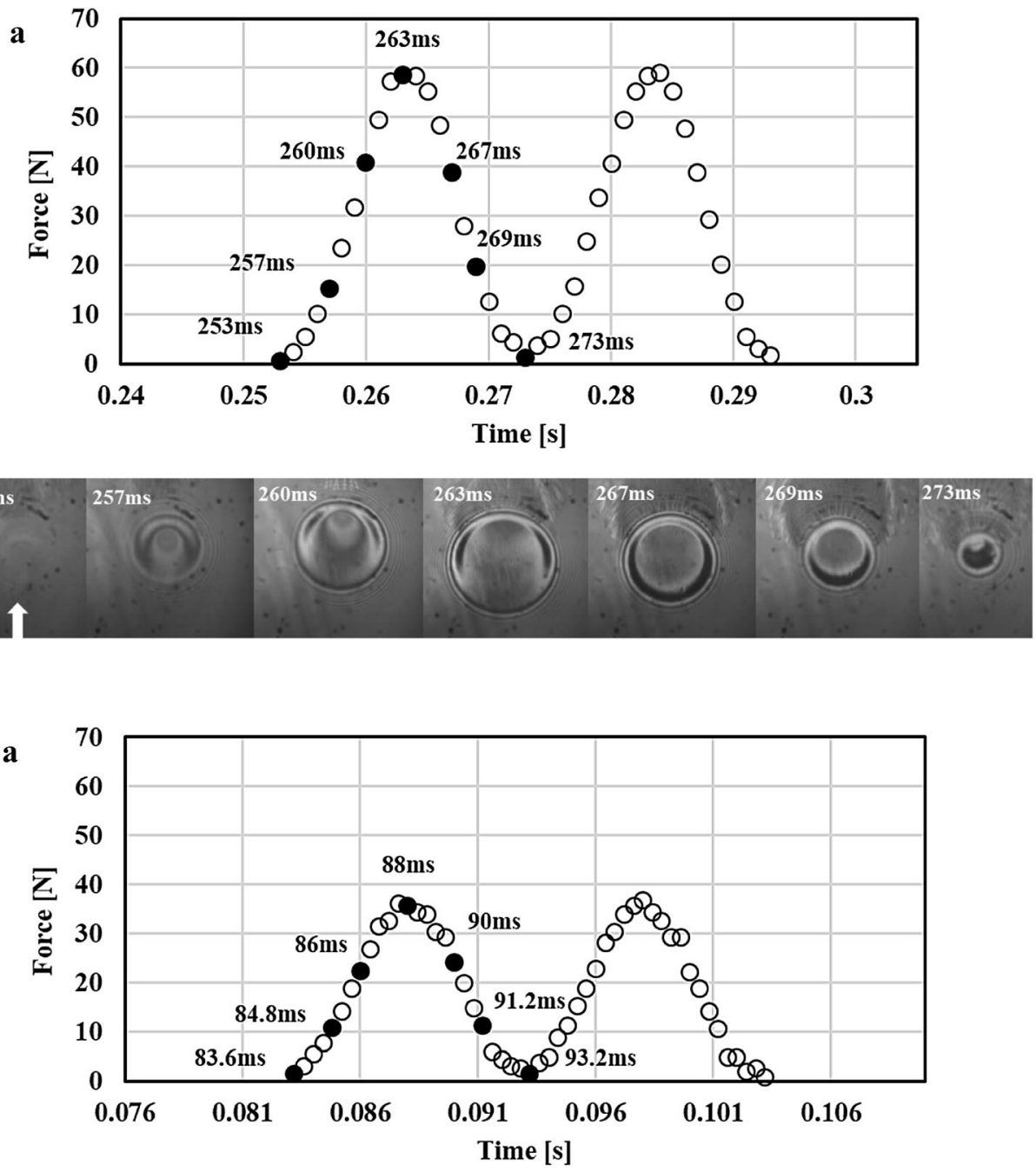

b

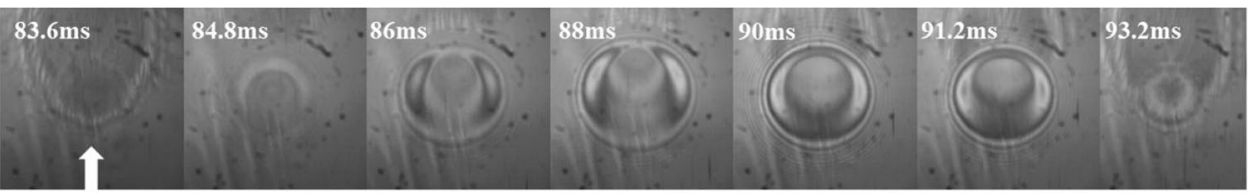

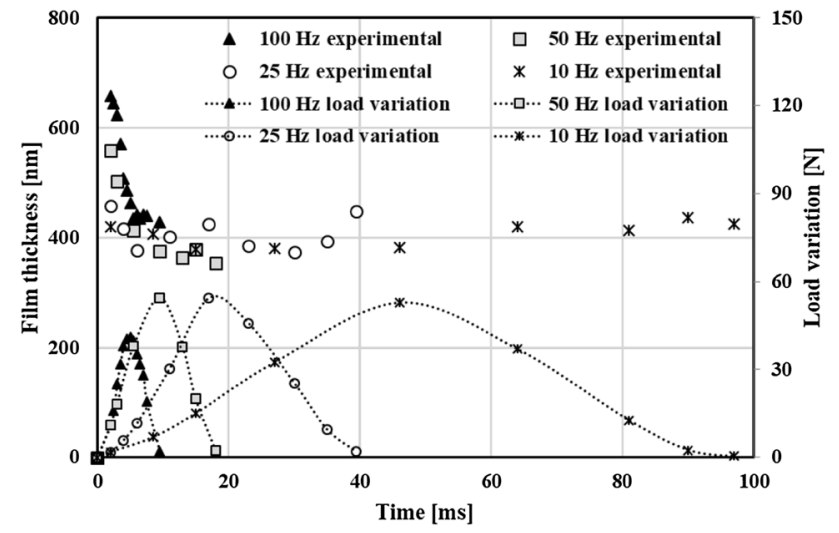

Fig. 7 Central film thickness at four frequencies, $0.05 \mathrm{~m} / \mathrm{s}$

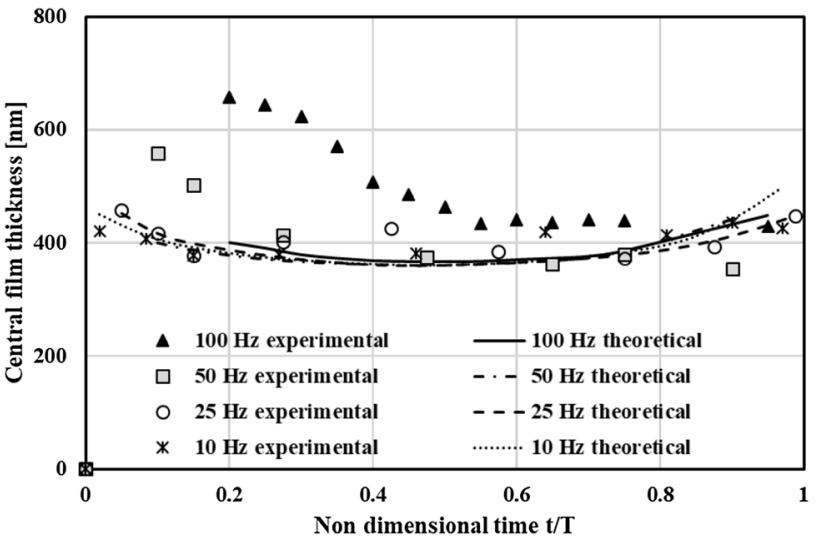

Fig. 8 Central film thickness variation over one cycle 


\subsection{Analytical Modelling}

The EHD film thickness is established by the conditions in the inlet of the contact, as mentioned above. In the current experiments, the entrainment velocity was kept constant at $50 \mathrm{~mm} / \mathrm{s}$. It is now assumed that the load varies between zero and a given value $2 P_{0}$ on a sinusoidal fashion, as expressed by Eq. (3).

$P(t)=P_{0}(1+\sin \omega t)$.

It is obvious that, during half a cycle, the contact size expands rapidly due to the variation of the load. At the inlet of the contact, this expansion is equivalent to an increase of the entrainment velocity during the load-increasing phase and a decrease during the decreasing of the load. As the contact edge moves in direction opposite to the lubricant entrainment direction, the lubricant approaches the convergent gap at a velocity equal to the sum of the velocity of the contact edge and the mean velocity of the surfaces. It results in a net (effective) entrainment velocity larger than that corresponding to steady-state conditions. According to Hertz's theory of elastic contact the radius of contact between a sphere and a plane depends of the load, the radius of the sphere and the elastic modulus of the contacting bodies [38]. In order to evaluate the transient entrainment speed, the radius of the contact is written as a function of time as shown in Eq. (4).

$a=\left(\frac{3 R}{4 E^{*}}\right)^{1 / 3} P(t)^{1 / 3}$.

The speed of change of the contact radius is $\mathrm{da} / \mathrm{dt}$, so combining (3) and (4) we get

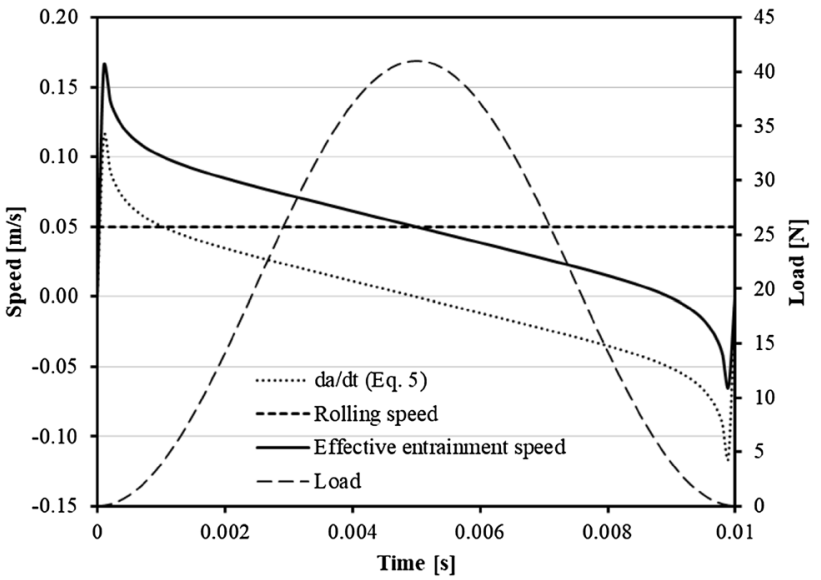

Fig. 9 Entrainment velocity in transient load conditions

the effective entrainment speed is over three times the rolling speed, which obviously would affect the EHD film thickness. In order to evaluate the transient film thickness, the formula given by Eq. (1) is written as

$h_{c}=A \cdot u^{2 / 3} P(t)^{-2 / 30}$.

In Eq. (1), 0.67 was approximated by $2 / 3$ and 0.067 by $2 / 30$, while $\mathrm{A}$ is a constant factor which includes all parameter except velocity and load. According to the above analysis, the effective entrainment velocity is equal to the rolling velocity and the velocity of change of the contact radius; the transient film thickness can thus be written as

$h(t)=A \cdot\left(u_{0}+\frac{d a}{d t}\right)^{2 / 3} P(t)^{-2 / 30}$,

$\frac{\mathrm{d} a}{\mathrm{~d} t}=\frac{\mathrm{d} a}{\mathrm{~d} P} \frac{\mathrm{d} P}{\mathrm{~d} t}=\frac{1}{3}\left(\frac{3 R}{4 E^{*}}\right)^{1 / 3} P(t)^{-2 / 3} P_{0} \omega \cos \omega t=\left(\frac{R P_{0}}{36 E^{*}}\right)^{1 / 3} \frac{\omega \cos \omega t}{(1+\sin \omega t)^{2 / 3}}$.

This relationship shows that the speed is different in the load-increasing and load-decreasing phases, as the functions sine and cosine are out of phase by $T / 4$. Another observation is that the speed becomes infinite when sinus equals -1 , that is, when the load is zero. Obviously, in reality the film thickness cannot be zero, and to avoid this, the entrainment velocity was set to zero when the load was zero. This is illustrated in Fig. 9 which shows the velocity of change of the contact radius, the set, rolling speed and the resultant or effective entrainment velocity. The load amplitude was taken as $20.5 \mathrm{~N}$ to match the experimental values for the $100 \mathrm{~Hz}$ tests.

It can be seen that, as a result of the harmonic load variation, the effective entrainment velocity is larger than the rolling speed of the surfaces during the load-increasing phase and smaller during the load-decreasing phase. At its peak, where $u_{0}$ is the constant rolling speed. If $u_{0}$ is brought in front of the bracket and taking into account Eq. (5), the timedependent film thickness becomes

$h(t)=A \cdot u_{0}^{2 / 3}\left[1+\frac{1}{u_{0}}\left(\frac{R P_{0}}{36 E^{*}}\right)^{1 / 3} \frac{\omega \cos \omega t}{(1+\sin \omega t)^{2 / 3}}\right]^{2 / 3} P(t)^{-2 / 30}$.

Using (6), the ratio of transient to steady-state film thickness (EHD film thickness calculated with formula (1) but with the load varying by (3) at each time step) can be written as

$\frac{h(t)}{h_{s t}}=\left[1+\frac{1}{u_{0}}\left(\frac{R P_{0}}{36 E^{*}}\right)^{1 / 3} \frac{\omega \cos \omega t}{(1+\sin \omega t)^{2 / 3}}\right]^{2 / 3}$. 
The variation of this ratio, together with the normalised load (current load/ maximum load) as a function of nondimensional time (time/period of load cycle), is shown in Fig. 10. The parameters of this simulation are frequency $100 \mathrm{~Hz}$, load-variation amplitude $20.5 \mathrm{~N}$ and entrainment speed $0.05 \mathrm{~m} / \mathrm{s}$. This graph shows that at the beginning of the load-increasing phase the transient film thickness is more than two times larger than the theoretical film thickness. As the load increases towards its maximum value, the transient film thickness tends towards the steady-state value.

It can be seen that, at a certain moment during the cycle, the film thickness becomes zero due to the fact that the effective entrainment velocity momentarily becomes zero as seen in Fig. 9. A pertinent question can be asked for the variation of the film thickness over the whole cycle: why are the values of the film thickness at the beginning and the end of the cycle not equal? The answer is that they are, but because they are infinite they cannot be shown in the graph. At the beginning of the cycle, the load is zero and obviously in this case Hamrock and Dowson Eq. (1) predicts infinite film, or rather no film.

\subsection{The Effect of Working Parameters}

The effect of the working parameters can be evaluated from relationship (9). The larger the frequency, the quicker the load increases and consequently the effective entrainment speed. Figure 11 shows the central film thickness for four frequencies at constant load amplitude of $20.5 \mathrm{~N}$ and a set entrainment speed of $0.05 \mathrm{~m} / \mathrm{s}$.

It can be seen that at lower frequencies the film thickness is almost equal to the steady-state value over the whole vibration cycle, while at the largest frequency employed in these tests, $100 \mathrm{~Hz}$, the transient formula predicts a film

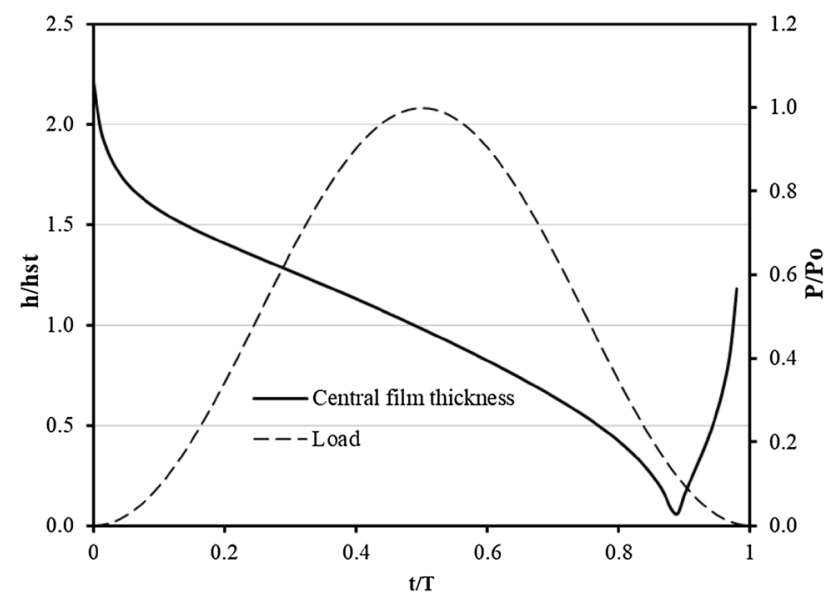

Fig. 10 Ratio of transient film thickness and steady-state film thickness at $100 \mathrm{~Hz}$

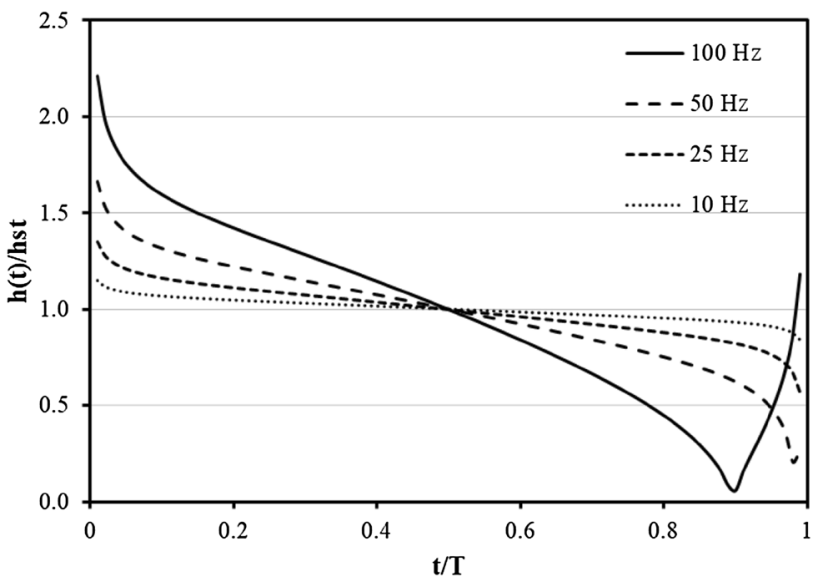

Fig. 11 The effect of frequency upon central film thickness

thickness 2.3 times larger than steady state at the beginning of the vibration cycle.

Figure 12 shows the variation of the maximum film thickness with the frequency of load variation.

Hooke and Morales-Espejel [39] performed a theoretical analysis of line contacts subjected to load fluctuations, on a non-zero mean cycle. The conditions of the current experiments are obviously quite different from those in [39]; however, it is interesting to make a similar evaluation of the effect of frequency upon maximum film thickness variation. The same non-dimensional parameters for wavelength, $\lambda / b P^{1.5} S^{-2}$, and maximum film thickness variation, $\delta h E^{\prime} / \delta F P^{0.75} S^{-1}$, with $\lambda=u / f, P=\alpha P_{\mathrm{H}}$, and $S=G U^{0.25}$ are used. In these, $u$ is the mean surface velocity, $f$ is the frequency, $\alpha$ is the pressure-viscosity coefficient, $P_{\mathrm{H}}$ is the Hertzian pressure, $G=\alpha E^{\prime}$ is the material parameter and $U=\left(\eta_{0} u / E^{\prime} R\right)$. The conditions used in current tests give the Greenwood pressure parameter $P$ of 14.2 and Greenwood

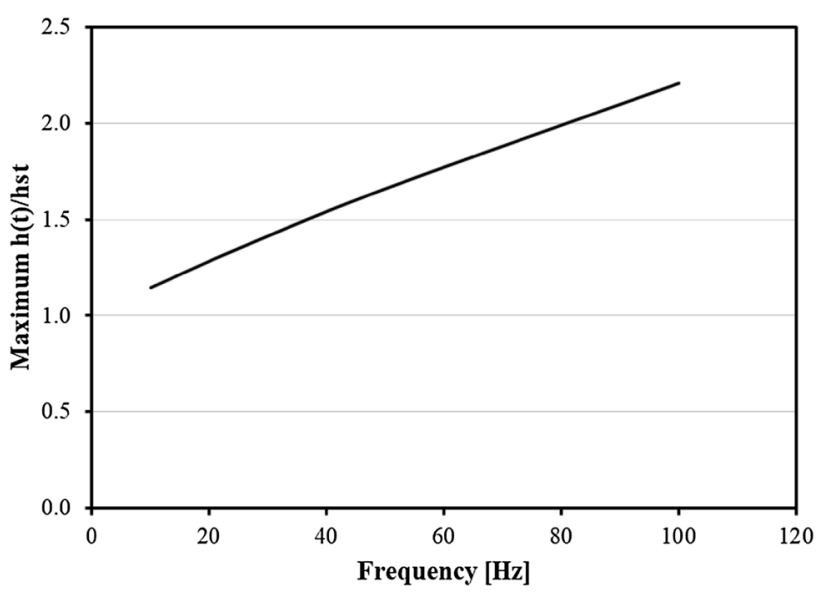

Fig. 12 The variation of maximum transient film thickness with frequency 
speed parameter $S=6.8$ and an inlet piezoviscous parameter $c=0.967 P^{0.2} S^{0.8}$ of 7.6. In [39], film thickness variation is given for values of $P=20$ and $S=8$, while $c$ ranged between 2 and 20. The non-dimensional film thickness versus nondimensional wavelength, for the four frequencies employed in these tests is shown in Fig. 13. The trend of this variation is very similar to that seen in Fig. 3a of Ref. 39, even accounting for the differences in the conditions used. It is obvious that the frequencies employed in the current tests are small enough such that the maxima of film thickness seen in [39] is not reached.

Another parameter which strongly influences the transient behaviour of EHD films is the entrainment speed of the contact. This effect is shown in Fig. 14 where the frequency was kept constant at $100 \mathrm{~Hz}$ and the entrainment speed (constant, set rolling speed, independent of the load variation) changes as seen. It is obvious that an increase of entrainment speed is equivalent to a decrease of the frequency.

Finally, the other parameter which influences the film thickness, in transient conditions, is the load amplitude. Figure 15 shows this effect for a contact running at the same frequency of $100 \mathrm{~Hz}$ and an entrainment speed set at $0.05 \mathrm{~m} / \mathrm{s}$.

It is known that the effect of the load upon the steadystate film thickness is almost negligible, in comparison to the effect of speed for example; however as seen in Fig. 15 in case of transient films, this parameter influences the film thickness as strongly as the entrainment speed. This is because by keeping the frequency constant, the change of the amplitude of the load is equivalent to a more rapid variation of contact dimensions, and thus the entrainment speed increases, according to Eq. (7).

\subsection{Comparison of Experiment and Theory}

This theoretical analysis was intended to give a qualitative and not quantitative insight to the behaviour of film

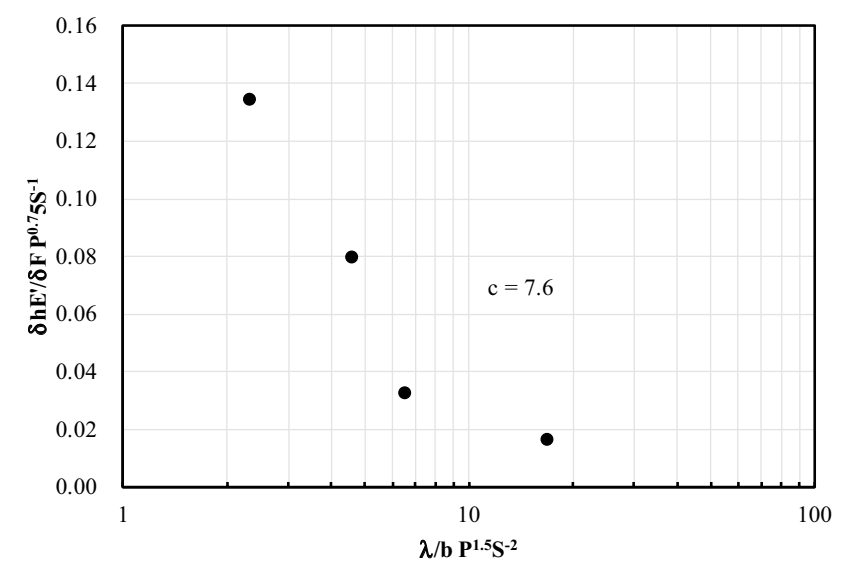

Fig. 13 Non-dimensional film thickness variation with non-dimensional wavelength

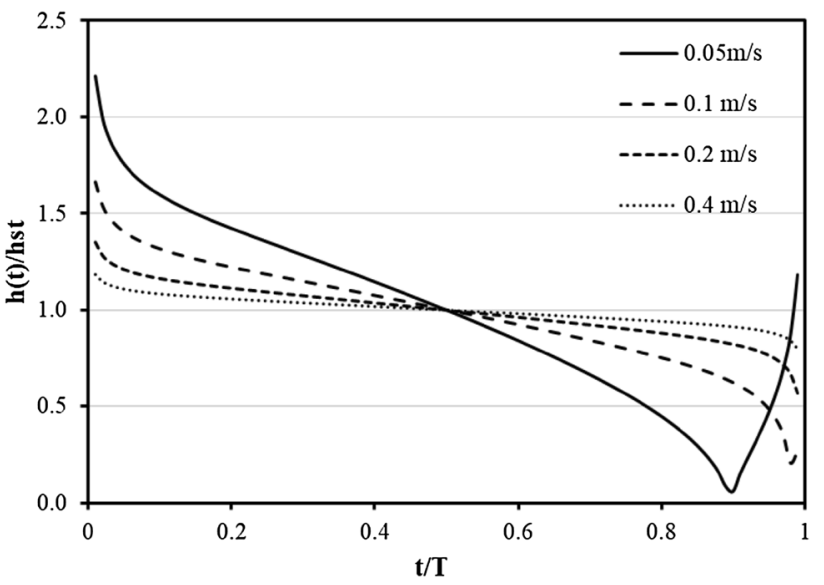

Fig. 14 The effect of entrainment speed upon transient film thickness

thickness under cyclically load variation; however, it is interesting to make a direct comparison to experimental values of the film thickness. Figure 16 shows such comparison. Obviously, the experimental values show a descending trend, like the theoretical curve, and reasonably agree with the later for a part of the cycle. It is clear that at the beginning and end of the load-variation cycle, where the effective entrainment velocity is largest and thus the squeeze effect is expected to be strongest, the measured values differ from the theoretical ones. The difference is especially great during the second half of the cycle, where the rapid decrease of the load makes the effective entrainment velocity drop rapidly and change sign, thus making the theoretical film thickness momentarily zero. At this moment, the experimental film thickness in the central region of the contact does not follow the speed and maintains large values due to the fact that the fluid cannot be squeezed out of the contact quickly enough.

As mentioned previously, squeeze and the time taken for the lubricant to travel across the contact are phenomena not

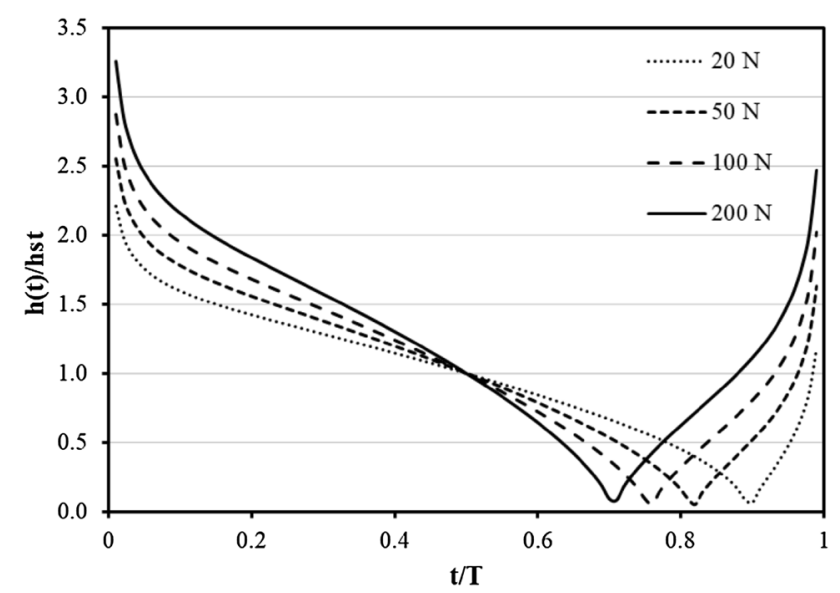

Fig. 15 Effect of load amplitude upon transient film thickness 


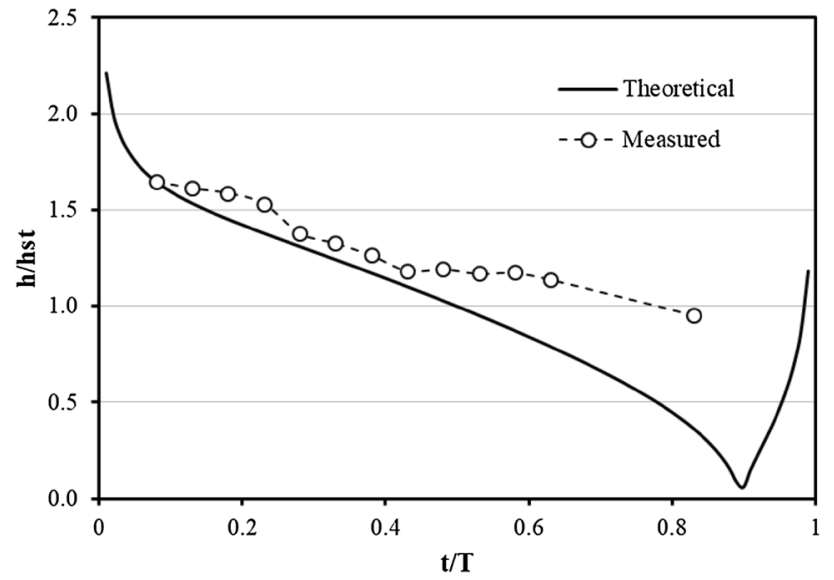

Fig. 16 Comparison between theoretical and experimental central film thickness $(50 \mathrm{~Hz}, 20.5 \mathrm{~N}$ amplitude, $0.05 \mathrm{~m} / \mathrm{s}$ )

taken into account in this theoretical analysis. The squeeze effect during the rapid decrease of the velocity (and the film thickness with it) results into a film larger than the steadystate predictions, by the formation of a lubricant entrapment, as proved experimentally and theoretically [40-43].

The current approach and results are supported by the results of the analytical/numerical investigation carried out in $[32,33]$, which conclude that between the increase of speed due to rapid increase of contact dimensions and the squeeze effects the former is dominant.

This qualitative analysis is evidently simplified and does not take into account the squeeze effect and the time of passage of the lubricant through the contact; however it is able to explain, at least partially, the behaviour of the film under cyclically variable loading.

\section{Conclusions}

Optical interferometry technique was used to measure the film thickness in an elastohydrodynamic contact, formed between a glass disc and a steel ball, subjected to harmonic variation of load. Apart from the ball and disc, the system comprised a white light source, a high-speed CCD camera, an electrodynamic shaker and a load cell for measuring the load applied to the contact. Interferometry images of the contact under vibrations were captured, recorded and analysed for film thickness over the contact area.

It was found that the lubricant central film thickness is significantly larger than that corresponding to steady-state application of the load during the load-increasing phase, about $60 \%$ larger for the conditions of these tests. During the load-decreasing phase, the central film thickness drops to values near and slightly below the steady-state condition.
A theoretical, analytical modelling of the film thickness behaviour during harmonic variation of load was carried out, based on the theoretical film thickness formula of Hamrock and Dowson. It was shown that the rapid increase of the contact dimensions is equivalent, in the inlet of the contact, with an increase of the effective entrainment speed. Consequently, the film thickness is larger than the steady-state predictions during the load-increasing phase and smaller during the load-decreasing phase. During the load-decreasing phase, the theoretical, transient film thickness momentarily falls to zero, at large frequencies and rather low set entrainment speed, although the measured results did not show this behaviour. This was explained by the squeeze effect, which prevents the lubricant to escape too quickly from the contact during the rapid decrease of the effective entrainment speed, and the formation of a lubricant entrapment.

Analyses of the effect of frequency, load amplitude and set entrainment speed were carried out, revealing that the frequency and amplitude of the load cycle have an opposite effect to the set entrainment speed.

The current paper provides a simple theoretical tool for prediction of lubricant film thickness in elastohydrodynamic contacts subjected to cyclic or non-cyclic variation of load. Unlike other approaches to this problem, the present analysis does not involve sophisticated numerical analysis tools.

Open Access This article is distributed under the terms of the Creative Commons Attribution 4.0 International License (http://creativeco mmons.org/licenses/by/4.0/), which permits unrestricted use, distribution, and reproduction in any medium, provided you give appropriate credit to the original author(s) and the source, provide a link to the Creative Commons license, and indicate if changes were made.

\section{References}

1. Cameron, A.: Righting a 40-year-old wrong. Tribol. Int. 18, 92 (1985)

2. Cameron, A.: Principles of Lubrication. Longmans, London (1966)

3. Gohar, R., Cameron, A.: Optical measurement of oil film thickness under elastohydrodynamic lubrication. Nature 200, 458-459 (1963)

4. Gohar, R., Cameron, A.: The mapping of elastohydrodynamic contacts. ASLE Trans. 10, 215-225 (1967)

5. Greenwood, J.A.: An extension of the Grubin theory of lubrication. J. Phys. D Appl. Phys. 5, 2195-2221 (1972)

6. Archard, J.F., Kirk, M.T.: Lubrication of point contacts. Proc. R. Soc. Ser. A 261, 532-550 (1961)

7. Cameron, A., Gohar, R.: Theoretical and experimental studies of the oil film in lubricated point contact. Proc. R. Soc., Ser. A 291, 520-536 (1966)

8. Dowson, D., Higginson, G.R.: Elastohydodynamic Lubrication, The Fundamentals of Roller and Gear Lubrication. Pergamon Press, London (1966) 
9. Hamrock, B.J., Dowson, D.: Isothermal elastohydrodynamic lubrication of point contact: Part 3. fully flooded results. Trans. ASME J. Lubr. Technol. 99, 264-276 (1977)

10. Chittenden, R.J., Dowson, D., Dunn, J.E., Taylor, C.M.: A theoretical analysis of the isothermal elastohydrodynamic lubrication of concentrated contacts. I: direction of lubricant entrainment coincident with the major axis of the contact ellipse. Proc. R. Soc. Lond. A397, 345-369 (1985)

11. Smeeth, M., Spikes, H.A.: Central and minimum elastohydrodynamic film thickness at high contact pressure. J. Tribol. 119(2), 291-296 (1997)

12. Hartl, M., Krupka, I., Poliscuk, R., Liska, M., Molimard, J., Querry, M., Vergne, P.: Thin film colorimetric interferometry. Tribol. Trans. 44(2), 270-276 (2001)

13. Glovnea, R.P., Olver, A.V., Spikes, H.A.: Experimental investigation of the effect of speed and load on film thickness in EHD contacts. Tribol. Trans. 48(2), 328-335 (2005)

14. Guanteng, G., Cann, P.M., Spikes, H.A., Olver, A.V.: Mapping of surface features in the thin film lubrication regime. In: Dowson D. (ed.) Proceedings of the 1998 Leeds Lyon Tribol. Symp. Elsevier Tribology Series 36, pp. 175-183 (1999)

15. Kaneta, M., Nishikawa, H.: The effects of a transversely oriented bump on point contact EHL films in reciprocating motion with a short length stroke. In: Dowson, D. (ed.) Lubrication at the Frontier, pp. 185-192. Elsevier B.V., New York (1999)

16. Choo, J.W., Glovnea, R.P., Olver, A.V., Spikes, H.A.: The effect of 3D model surface roughness features on lubricant film thickness in EHL contacts. ASME Trans. J. Tribol. 125(3), 533-542 (2003)

17. Wang, X., Kato, K., Adachi, K., Aizawa, K.: Load carrying capacity map for the surface texture design of $\mathrm{SiC}$ thrust bearing sliding in water. Tribol. Int. 36, 189-197 (2003)

18. Mourier, L., Mazuyer, D., Lubrecht, A.A., Donnet, C.: Transient increase of film thickness in micro-textured EHL contacts. Tribol. Int. 39, 1745-1756 (2006)

19. Krupka, I., Hartl, M.: The effect of surface texturing on thin EHD lubrication films. Tribol. Int. 40, 1100-1110 (2006)

20. Hooke, C.J.: The minimum film thickness in line contacts during reversal of entrainment. J. Tribol. 115, 185-192 (1993) 191-199

21. Sugimura, J., Jones, W.R., Jr., Spikes, H.A.: EHD film thickness in non-steady state contacts. Trans. ASME J. Tribol. 120, 442-452 (1998)

22. Glovnea, R.P., Spikes, H.A.: The influence of lubricant on film collapse rate in high pressure thin film behaviour during sudden halting of motion. STLE Tribol. Trans. 43(4), 731-739 (2000)

23. Holmes, M.J.A., Evans, H.P., Snidle, R.W.: Comparison of transient EHL calculations with shut-down experiments. Tribol. Interface Eng. Ser. 41, 79-89 (2003)

24. Glovnea, R.P., Spikes, H.A.: Elastohydrodynamic film formation at the start-up of the motion. Proc. Inst. Mech. Eng. J. Eng. Tribol. 215, 125-138 (2001)

25. Popovici, G., Venner, C.H., Lugt, P.M.: Effects of load system dynamics on the film thickness in EHL contacts during start-up. ASME Trans. J. Tribol. 126, 258-266 (2004)
26. Larsson, R., Lundberg, J.: Observations in transiently loaded EHL contacts under pure sliding conditions. STLE Tribol. Trans. 41(4), 489-496 (1998)

27. Wijnant, Y.H., Venner, C.H., Larsson, R., Eriksson, P.: Effects of structural vibrations on the film thickness in an EHL circular contact. J. Tribol. 121, 259-264 (1999)

28. Kaneta, M., Ozaki, S., Nishikawa, H., Guo, F.: Effects of impact loads on point contact elastohydrodynamic lubrication films. Proc. Inst. Mech. E. J. Eng. Tribol. 221, 271-278: (2007)

29. Rao, S.S.: Mechanical Vibrations. Pearson, Upper Saddle River (2016)

30. El Kilali, T., Perret-Liaudet, J., Mazuyer, D.: Experimental analysis of a high pressure lubricated contact under dynamic normal excitation force. Transient Process. Tribol. 43, 409-418 (2004)

31. Sakamoto, M., Nishikawa, H., Kaneta, M.: Behavikaor of point contact EHL films under pulsating loads. Trans. Proc. Tribol. 43, 391-399 (2004)

32. Morales-Espejel, G.E.: Central film thickness in time-varying normal approach of rolling elastohydrodynamically lubricated contacts. In: Proceedings of the IMechE Part C: J. Mech. Eng. Sci., vol. 222 (2008)

33. Felix-Quinonez, A., Morales-Espejel, G.E.: Film thickness fluctuations in time-varing normal loading of rolling elastohydrodynamically lubricated contacts. In: Proceedings of the IMechE Part C: J Mech. Eng. Sci., vol. 224 (2010)

34. Johnston, G.J., Wayte, R., Spikes, H.A.: The measurement and study of very thin lubricant films in concentrated contacts. Tribol. Trans. 34(2), 187-194 (1991)

35. Cann, P.M., Spikes, H.A., Hutchinson, J.: The development of a spacer layer imaging method (SLIM) for mapping elastohydrodynamic contacts. Tribol. Trans. 39(4), 915-921 (1996)

36. Marklund, O., Gustafsson, L.: Interferometry-based measurements of oil-film thickness. Proc. Inst. Mech. Eng. J 215, 243-259 (2001)

37. Zhang, X.: The effect of vibrations on the behaviour of elastohydrodynamic contacts. PhD thesis, University of Sussex: (2017)

38. Johnson, K.L.: Contact Mechanics. Cambridge: Cambridge University Press (1985)

39. Hooke, C.J., Morales-Espejel, G.E.: The effect of small sinusoidal load variations in elastohydrodynamic line contacts. ASME. J. Tribol. 138, 031501-031509 (2016)

40. Yang, P.R., Wen, S.Z.: Pure squeeze action in an isothermal elastohydrodynamic lubricated spherical conjunction. Part 1: Theory and dynamic load results. Wear 142, 1-16 (1991)

41. Glovnea, R.P., Spikes, H.A.: Elastohydrodynamic film collapse during rapid deceleration-Part I: experimental results. ASME Trans. J. Tribol. 123(2), 254-261 (2001)

42. Glovnea, R.P., Spikes, H.A.: Elastohydrodynamic film collapse during rapid deceleration-Part II: theoretical analysis and comparison of theory to experiment. ASME Trans. J. Tribol. 123(2), 262-267 (2001)

43. Guo, F., Nushikawa, H., Yang, P., Kaneta, M.: EHL under cyclic squeeze motion. Tribol. Int. 40, 1-9 (2007) 